\title{
THE VANISHING NEIGHBOURHOOD OF NON-ISOLATED SINGULARITIES
}

\author{
MIHAI TIBĂR
}

\begin{abstract}
We study the vanishing neighbourhood of non-isolated singularities of functions on singular spaces by associating a general linear function. We use the carrousel monodromy in order to show how to get a better control over the attaching of thimbles. For one dimensional singularities, we prove obstructions to integer (co)homology groups and to the eigenspaces of the monodromy via monodromies of nearby sections. Our standpoint allows to find, in certain cases, the structure of the Milnor fibre up to the homotopy type.
\end{abstract}

\section{INTRODUCTION}

In the landscape of singularities of holomorphic functions, the non-isolated singularities play a particular role. Their study has been initiated in the '70 and ' 80 by Y. Yomdin, R. Randell and Lê D.T. As a natural first extension of isolated singularities, the case of 1-dimensional singularities got special attention. The first results about the homotopy type of the Milnor fibre were proved by D. Siersma [Si1] and his studies opened the way to a series of other results for 1, 2 or higher dimensional singularities, by R. Pellikaan, T. de Jong, A. Zaharia, A. Némethi, G. Jiang, J. Fernandez and others. Meanwhile, the progress in stratified Morse theory by Goresky and MacPherson allowed one to treat singular holomorphic functions on singular spaces. The viewpoint due to Lê D.T. which consists in associating to the function $f$ a general linear function $l$ and studying the couple $(l, f)$ gave rise to new insight in the topic of non-isolated singularities. In particular, the study of the "box" neighbourhood lead to new results more recently, which exploit further the properties of the monodromies appearing in the fibration defined by $(l, f)$ (cf D. Siersma, J. Steenbrink, M. Saito, D. Massey and others). There are some other streams of research, which we shall not mention here.

Let $f:(X, 0) \rightarrow \mathbb{C}$ be a holomorphic function defined on the germ $(X, 0)$ of a singular space of pure dimension $n+1$, embedded into $\left(\mathbb{C}^{m}, 0\right)$ for some $m$. We work under the following technical but natural condition: "the rectified homotopical depth of $X$ is maximal", which includes the cases $X$ is smooth or a complete intersection. Our aim is to find how the Milnor fibre $F$ of $f$ is build from ingredients associated to restrictions of $f$ to lower dimensional slices of the space, which have therefore lower dimensional singularities.

It is well known that the Milnor fibre $F$ of $f$ is $(n-k)$-connected, where $k$ denotes the dimension of the stratified singular locus of $f$. This follows by a Lefschetz-type argument, from comparing $F$ to the Milnor fibre $F^{\prime}$ of the restriction $f_{l l=0}$, where $l$ is

2000 Mathematics Subject Classification. 32S55, 32S50, 32S40, 32S99.

Key words and phrases. functions on singular spaces, non-isolated singularities, Milnor fibre, monodromy. 
general enough. We go beyond this comparison and relate $F$ to $F^{\prime}$ via a slice $\{l=\eta\}$ near to the origin (Theorem 2.2): $H_{n}(F)$ and $H_{n-1}(F)$ are the kernel and respectively the cokernel of a certain morphism $\tilde{H}_{n}\left(F \cup F_{D}^{\prime}\right) \rightarrow H_{n}\left(F_{D}^{\prime}, F^{\prime}\right)$, see Figure 1 for a rapid location. This comparison allows us to exploit the $l$-monodromy and deduce bounds for the betti numbers $b_{n-1}(F)$ and $b_{n}(F)$. The pair $\left(F_{D}^{\prime}, F^{\prime}\right)$ has the advantage that it can further be localised, by excision, in a tubular neighbourhood of the slice $\operatorname{Sing} f \cap F_{D}^{\prime}$ of the singular locus.

We then specialise to the case $\operatorname{dim} \operatorname{Sing} f=1$. Here we show how the $l$-monodromy is related to the "vertical monodromy" of the transversal singularities of a slice near to the origin. We get more explicit bounds for $b_{n-1}(F)$ and for the eigenspaces and the maximal Jordan blocks of the monodromy, as well as divisibility results for the characteristic polynomial of the monodromy $h$, in terms of transversal singularities. These extend to a more general setting some of Siersma's results proved in Si2]. Our proof is based on the controlled attaching of cells, whereas Siersma's proof uses special variation maps.

We finally show how our standpoint allows to recover and throws a new light on Siersma's bouquet result for "line singularities" (Corollary 4.3). Further developments are in progress as a joint work with Dirk Siersma [ST].

\section{A GeOMETRIC VIEWPOINT}

Let $(X, 0)$ be a germ of a singular space of pure dimension $n+1$ and let $f:(X, 0) \rightarrow \mathbb{C}$ be a holomorphic function. We shall assume that our space $(X, 0)$ satisfies one of the following two conditions (where the former implies the latter). We refer to $\mathrm{HL}$ for the notions of rectified homotopical depth $r h d(X)$ and rectified homological depth $r H d(X)$.

(*) $\operatorname{rhd}(X) \geq \operatorname{dim}_{0} X$, respectively

(**) $r H d(X) \geq \operatorname{dim}_{0} X$.

These conditions are both true in case $X$ is a complete intersection (arbitrarily singular). It was proved by Hamm and Lê $\mathrm{HL}$ that condition $(*)$, resp. (**), implies that the complex link of $(X, p)$, for any $p$, is homotopically equivalent to a bouquet of $n$-spheres, respectively has the reduced homology concentrated in dimension $n$. These also imply that any function with a stratified Morse singularity at some point of $X$ has Milnor fiber homotopy equivalent to a bouquet of $n$-spheres, resp. with reduced cohomology concentrated in dimension $n$. Condition $r H d(X) \geq n+1$ is further equivalent to the fact that the constant sheaf on $X$ is perverse.

We denote by Sing $\phi$ the singular locus of some holomorphic map $\phi:(X, 0) \rightarrow\left(\mathbb{C}^{p}, 0\right)$, defined as the closure of the union of the singular loci Sing $\phi_{\mid W_{i}}$ of the restriction of $\phi$ to the strata $W_{i}$ of some Whitney stratification $\mathcal{W}$ of $X$ which we fix throughout the paper.

Let us chose a generic linear function $l:(X, 0) \rightarrow \mathbb{C}$ and let $B_{\varepsilon}$ denote a Milnor ball for $f$, that is the intersection of a small enough ball at the origin of the ambient space with a suitable representative of the germ $(X, 0)$. Lê D.T. showed (see e.g. Lê2]) that one can use the map $(l, f): B \rightarrow \mathbb{C}^{2}$ and a neighbourhood "box" $B:=B_{\varepsilon} \cap l^{-1}\left(D_{\eta^{\prime}}\right) \cap f^{-1}\left(D_{\gamma^{\prime}}\right)$ in order to describe the local Milnor fibration of $f$ and its relation to the Milnor fibration of the slice $f_{\mid l=0}$. We have already used this approach in Ti1, Ti2] in order to get control over 
the attaching of cells via the monodromy, in case of a function $f$ with isolated singularity. Let us set the notations and recall several facts.

If $l$ is general enough, then the polar locus $\Gamma:=\operatorname{closure}\{\operatorname{Sing}(l, f) \backslash\{f=0\}\}$ is a curve on $(X, 0)$, or it is empty. Each point of the intersections $B \cap \Gamma \cap l^{-1}(\eta)$ and of $B \cap \Gamma \cap f^{-1}(\gamma)$ is a stratified Morse singularity of the restrictions $f_{l^{-1}(\eta)}$ and $l_{f^{-1}(\gamma)}$ respectively. Let us denote by $\Delta:=(l, f)(\Gamma)$ the Cerf diagram in the target space $\mathbb{C}^{2}$. Take $\eta \ll \eta^{\prime}$ and $\gamma \ll \gamma^{\prime}$ such that the intersection $\left(D_{\eta} \times\{\gamma\}\right) \cap \Delta$ with the Cerf diagram $\Delta$ is contained in the interior of $D_{\eta} \times\{\gamma\}$. We use the following notations and remarks:

(1) $F:=B \cap f^{-1}(\gamma) \cap l^{-1}\left(D_{\eta}\right)$ is the Milnor fiber of $f$. Instead of the point $\gamma$ one can take any point on $\partial D_{\gamma}$.

(2) $F^{\prime}:=B \cap(l, f)^{-1}(\eta, \gamma)$ is homeomorphic to the Milnor fiber of the restriction $f_{\mid\{l=0\}}$ (but of course has a different monodromy over the circle $\{\eta\} \times \partial \bar{D}_{\eta}$ ).

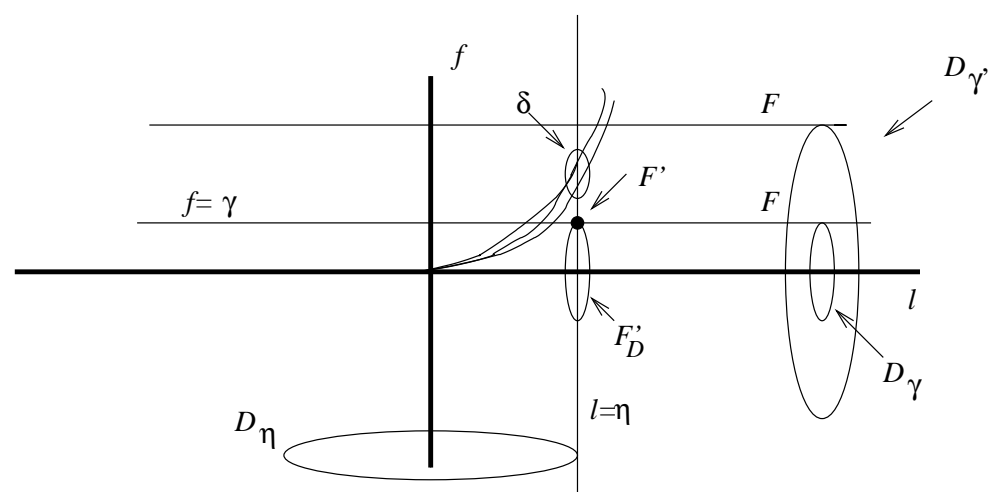

Figure 1. Cerf diagram

(3) $F_{D}:=B \cap f^{-1}\left(D_{\gamma}\right)$ is a Milnor tube, hence contractible.

(4) $F_{D}^{\prime}:=F_{D} \cap l^{-1}(\eta)$ retracts to the fibre $B \cap f^{-1}(0) \cap l^{-1}(\eta)$, which is the complex link of the hypersurface $f^{-1}(0)$ at the origin.

We collect below a bunch of results from the literature, which will play a key role in the following.

FACTS 2.1. (a) Under condition $(*)$, the complex link of $X$ at 0 is homotopy equivalent to a wedge of spheres $\vee S^{n}$. The complex link of the hypersurface $f^{-1}(0)$ at the origin, denoted $l \mathrm{k}\left(f^{-1}(0), 0\right)$, is homotopy equivalent to a wedge of spheres $\vee S^{n-1}$. This follows from the fact that $f^{-1}(0)$ inherits the property $\left(^{*}\right)$, see [HL, Th. 3.2.1]. Consequently $F_{D}^{\prime}$ is homotopy equivalent to a wedge of spheres $\vee S^{n-1}$, since $F_{D}^{\prime} \stackrel{\text { ht }}{\simeq} \operatorname{lk}\left(f^{-1}(0), 0\right)$.

(b) The complex link of $X$ at 0 , denoted $\operatorname{lk}(X, 0)$, is obtained by attaching to the complex link of $f^{-1}(0)$ at the origin, denoted $l \mathrm{k}\left(f^{-1}(0), 0\right)$, a number of cells, which are all of dimension $n$ if condition $(*)$ is assumed. Each cell corresponds to a point of intersection of the polar curve $\Gamma$ with the slice $l^{-1}(\eta)$, see [Lê1, Lê2, Ti1]. In Figure 1, this can be visualised as attaching to $F_{D}^{\prime}$ the cells corresponding to the intersection $\Gamma \cap l^{-1}(\eta)$. 
(c) There is a topological disk $\{\eta\} \times \delta$ containing all intersection points $\Delta \cap\{l=\eta\}$ and is disjoint from the disk $\{\eta\} \times D_{\gamma}$. This disk can be made sliding along the Cerf diagram to a zone $W \subset D_{\eta} \times\{\gamma\}$. This procedure, due to Lê D.T., is known as "rabattement dans le diagramme de Cerf" and was used in our bouquet structure theorem [Ti1] for the Milnor fibre of function germs $f$ with isolated singularity. So, revisiting the point (a) above, after attaching to $F_{D}^{\prime}$ the cells from the zone $(l, f)^{-1}(W)$, which we have identified to $(l, f)^{-1}(\{\eta\} \times \delta)$, we get the complex link of $X$, hence a bouquet of $n$-spheres. If we continue to attach the other $n$-cells coming from the intersection points of $\Gamma$ with $F$ which are outside $(l, f)^{-1}(W)$, then, as result, we can only get more $n$-spheres in the bouquet.

Let $h$ denote the monodromy on the (co)homology of the fibre of $f$. This is induced by a geometric monodromy which acts on $F$ and on $F^{\prime}$, and which we shall denote by the same symbol $h$. It acts as the identity on $F_{D}$ and on $F_{D}^{\prime}$.

There is also the action of the geometric $l$-monodromy: on $F_{D}, F$ and $F_{D}^{\prime}$ it is isotopic to the identity, but it may be non-trivial on $F^{\prime}$.

Theorem 2.2. Let $(X, 0)$ satisfy the condition $\left({ }^{* *}\right)$ and let $f:(X, 0) \rightarrow \mathbb{C}$ be any holomorphic function germ. Then the following sequences are exact:

$$
0 \rightarrow \tilde{H}_{n}(F) \rightarrow \tilde{H}_{n}\left(F \cup F_{D}^{\prime}\right) \rightarrow H_{n}\left(F_{D}^{\prime}, F^{\prime}\right) \rightarrow \tilde{H}_{n-1}(F) \rightarrow 0
$$

and

$$
0 \rightarrow \tilde{H}^{n-1}(F) \rightarrow H^{n}\left(F_{D}^{\prime}, F^{\prime}\right) \rightarrow \tilde{H}^{n}\left(F \cup F_{D}^{\prime}\right) \rightarrow \tilde{H}^{n}(F) \rightarrow 0
$$

and there are natural isomorphisms for all $j \geq 1$ :

$$
\tilde{H}_{n-j-1}(F) \simeq H_{n-j}\left(F_{D}^{\prime}, F^{\prime}\right) \text {, resp. } \tilde{H}^{n-j-1}(F) \simeq H^{n-j}\left(F_{D}^{\prime}, F^{\prime}\right) .
$$

The monodromies $h$ and $l$ act on all these morphisms.

Proof. Let us first consider the union $F \cup F_{D}^{\prime}$, where $F \cap F_{D}^{\prime}=F^{\prime}$. Note that both the monodromies $h$ and $l$ are in general not isotopic to the identity on $F \cup F_{D}^{\prime}$. From Facts 2.1(b)(c) it follows that $\tilde{H}_{*-1}\left(F \cup F_{D}^{\prime}\right)=\tilde{H}_{*-1}\left(\vee S^{n}\right)$, and one can also deduce the exact number of spheres in the bouquet.

Since $F_{D}$ is contractible, we get $H_{*}\left(F_{D}, F \cup F_{D}^{\prime}\right)=\tilde{H}_{*-1}\left(F \cup F_{D}^{\prime}\right)$. The same is true in cohomology.

Consider next the the pair $\left(F \cup F_{D}^{\prime}, F\right)$. By excision we get the isomorphism:

$$
H_{*}\left(F \cup F_{D}^{\prime}, F\right) \stackrel{\widetilde{\leftarrow}}{\leftarrow} \tilde{H}_{*}\left(F_{D}^{\prime}, F^{\prime}\right),
$$

and its similar counterpart in cohomology. The geometric monodromies $h$ and $l$ act on all these sequences.

Let then consider the long exact sequence of the triple $\left(F_{D}, F \cup F_{D}^{\prime}, F\right)$. This splits into short sequences since the homology $H_{*}\left(F_{D}, F \cup F_{D}^{\prime}\right)$ is concentrated in dimension $n+1$. In order to complete the proof of our complete claim we just have to remark the isomorphism $H_{*}\left(F_{D}, F\right)=H_{*-1}(F)$. The proof in cohomology parallels the one in homology.

We derive the following bound for the betti number $b_{n}(F)$.

Corollary 2.3. $b_{n}(F) \leq \lambda^{0}+b_{n}(\operatorname{lk}(X, 0))$, where $\lambda^{0}=\operatorname{int}_{0}\left(\Gamma, f^{-1}(0)\right)-\operatorname{int}_{0}\left(\Gamma, l^{-1}(0)\right)$. 
Proof. This follows from the first map in the above theorem and the computation $\operatorname{dim} \tilde{H}_{n}(F \cup$ $\left.F_{D}^{\prime}\right)=\lambda^{0}+b_{n}(\operatorname{lk}(X, 0))$ explained in Facts 2.1(b)(c). Also remember that the (co)homology of $\operatorname{lk}(X, 0)$ is concentrated in dimension $n$, since condition $(* *)$ is assumed. Note that $\lambda^{0}$ depends only on $(X, 0)$ and $f$ but not on the choice of generic $l$.

If $X$ is nonsingular, then $\operatorname{lk}(X, 0)$ is acyclic and we recover the known well-known inequality $b_{n}(F) \leq \lambda^{0}$.

We further investigate the pair $\left(F_{D}^{\prime}, F^{\prime}\right)$. Let then $T$ denote a tubular neighbourhood of Sing $f \cap F_{D}^{\prime}$ within $F_{D}^{\prime}$. Therefore $T$ retracts to the complex link of Sing $f$ at the origin. ${ }^{1}$ Let $L$ denote the action on (co)homology of the $l$-monodromy. We get:

Corollary 2.4. (a) In Theorem 2.2 one may replace $H_{*}\left(F_{D}^{\prime}, F^{\prime}\right)$ by $H_{*}\left(T, T \cap F^{\prime}\right)$, and similarly in cohomology.

(b) coker $\left(L-\mathrm{id} \mid H_{n}\left(T, T \cap F^{\prime}\right)\right)$ surjects onto $H_{n-1}(F)$, respectively $H^{n-1}(F)$ injects into $\operatorname{ker}\left(L-\mathrm{id} \mid H^{n}\left(T, T \cap F^{\prime}\right)\right)$. Consequently $b_{n-1}(F) \leq \operatorname{dim} k e r\left(L-\mathrm{id} \mid H^{n}(T, T \cap\right.$ $\left.F^{\prime}\right)$ ).

Proof. (a) The substitution is due to the excision: $H_{*}\left(F_{D}^{\prime}, F^{\prime}\right) \stackrel{\widetilde{E}}{\leftarrow} H_{*}\left(T, T \cap F^{\prime}\right)$. Point (b) follows from (a) and from the exact sequence of Theorem 2.2 (a) on which the $l$-monodromy acts as follows. The action of the geometric $l$-monodromy on $F$ is isotopic to the identity. It is therefore the identity on the (co)homology of $F$ and of $\left(F_{D}, F\right)$.

REMARK 2.5. Since the geometric $l$-monodromy is the identity on $F$, it induces the identity on $H_{n-j}\left(F_{D}^{\prime}, F^{\prime}\right)$ and on $H_{n-j}\left(T, T \cap F^{\prime}\right)$ for all $j \geq 1$, by Theorem 2.2(b) and Corollary 2.4(a).

\section{3. $\mathrm{CASE} \operatorname{dim} \operatorname{Sing} f=1$}

We specialize to the case $\operatorname{dim} \operatorname{Sing} f=1$ and point out several consequences of the preceding results. We still assume in this section that $(X, 0)$ satisfies the condition $(* *)$. In case of $\operatorname{dim} \operatorname{Sing} f=1$, the tubular neighbourhood $T$ consists of small Milnor balls $B_{i}$ at the finitely many points $\operatorname{Sing} f \cap F_{D}^{\prime}$. Let $F_{i}$ denote the Milnor fiber of such an isolated singularity of the restriction of $f$ to the transversal slice $F_{D}^{\prime}$. Then the geometric monodromy $h$ on $F$ restricts to the Milnor monodromy $h_{i}$ of $F_{i}$, for each $F_{i}$. Let $L$ denote, as before, the action on (co)homology of the l-monodromy. The l-monodromy acts on the points Sing $f \cap F_{D}^{\prime}$ by certain permutations; each point comes back to itself after applying a number of times the l-monodromy. We denote by $\nu_{i}$ the action on the (co)homology of $F_{i}$ of the come-back monodromy ${ }^{2}$. One notes that $F_{i}$ as well as $\nu_{i}$ depend only on the component $\Sigma_{j}$ of $\operatorname{Sing} f$. We then get:

Proposition 3.1. If condition (**) holds and if $\operatorname{dim} \operatorname{Sing} f=1$ then:

$$
\operatorname{coker}\left(L-\mathrm{id} \mid H_{n}\left(T, T \cap F^{\prime}\right)\right) \simeq \oplus_{j} \operatorname{coker}\left(\nu_{j}-\mathrm{id} \mid H_{n-1}\left(F_{j}\right)\right)
$$

where the sum is taken over the components $\Sigma_{j}$ of $\operatorname{Sing} f$.

\footnotetext{
${ }^{1}$ In case $\operatorname{Sing} f$ is a complete intersection this is homotopy equivalent to a wedge of spheres of dimension $\operatorname{dim} \operatorname{Sing} f-1$.

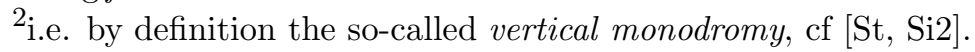


Proof. We observe that $H_{*}\left(T, T \cap F^{\prime}\right)$ is isomorphic, by excision, to $\oplus_{i} H_{*}\left(B_{i}, F_{i}\right)=$ $\oplus_{i} \tilde{H}_{*-1}\left(F_{i}\right)$, where the sum is taken over all the singular points which are in the linear slice $F_{D}^{\prime}$. As shown in [Si3] or [Ti2], there is a cyclic movement of a singular point belonging to some component $\Sigma_{j}$ of Sing $f$. This yields a particular shape of the matrix of $L$ : one can start with a basis $(e)$ of $H_{n-1}\left(F_{1}\right)$ and then $\left(L^{k}(e)\right)$ is a basis for $H_{n-1}\left(F_{k+1}\right)$, for all $k \in \overline{1, s_{j}-1}$, where $s_{j}$ is the number of points of intersection of a hyperplane slice $l=\eta$ with the component $\Sigma_{j}$ of Sing $f$. This gives the following direct sum splitting:

$$
\operatorname{coker}\left(L-\mathrm{id} \mid \oplus_{i} H_{n-1}\left(F_{i}\right)\right) \simeq \oplus_{j} \operatorname{coker}\left(\nu_{j}-\mathrm{id} \mid H_{n-1}\left(F_{j}\right)\right),
$$

where in the second sum we take one point for each component $\Sigma_{j}$ of $\operatorname{Sing} f$.

Corollary 3.2. (a) $b_{n-1}(F) \leq \operatorname{dim} \oplus_{\Sigma_{j}} \operatorname{coker}\left(\nu_{j}-\right.$ id $\left.\mid H_{n-1}\left(F_{j}\right)\right)$.

(b) $\operatorname{char}_{h \mid H_{n-1}(F)}$ divides $\prod_{\Sigma_{j}} \operatorname{char}_{h_{j} \mid \operatorname{coker}\left(\nu_{j}-\mathrm{id} \mid H_{n-1}\left(F_{j}\right)\right)}$.

In particular $\operatorname{char}_{h \mid H_{n-1}(F)}$ divides the product $\prod_{\Sigma_{j}} \operatorname{char}_{h_{j} \mid H_{n-1}\left(F_{j}\right)}$.

In case of non-singular $X$, this result was proved by Dirk Siersma [Si2] with a different proof. A weaker version, yet for singular $(X, 0)$ satisfying $(* *)$, was proved in [Ti2].

Proof. From Corollary 2.4(b) and Proposition 3.1] we deduce the surjection:

$$
\oplus_{j} \operatorname{coker}\left(\nu_{j}-\mathrm{id} \mid H_{n-1}\left(F_{j}\right)\right) \rightarrow H_{n-1}(F),
$$

from which (a) follows immediately.

(b). The monodromy $h$ acts on the preceding surjection. On the left hand side this amounts to the action of $\oplus_{j} h_{j}$. One remarks that the monodromies $h_{j}$ and $\nu_{j}$ commute. Then apply char $_{h}$ to this and get the claimed divisibility.

Let us remark that the analogous results are true in cohomology by standard reasons; one just replaces "surjection" by "injection" and "coker" by "ker".

Let $b_{\lambda}(V, \mu)$ denote the dimension of the eigenspace corresponding to the eigenvalue $\lambda$ of the linear operator $\mu$ acting on the vector space $V$. Let $J_{\lambda}(V, \mu)$ denote the maximum of the sizes of the Jordan blocks. With these notations we have:

Corollary 3.3. In cohomology, let $K_{j}:=\operatorname{ker}\left(\nu_{j}-\mathrm{id} \mid H^{n-1}\left(F_{j}\right)\right)$. Then:

(a) $H^{n-1}(F) \subset \oplus_{\Sigma_{j}} K_{j}$.

(b) $b_{\lambda}\left(H^{n-1}(F), h\right) \leq \sum_{\Sigma_{j}} b_{\lambda}\left(K_{j}, h_{j}\right)$.

(c) $J_{\lambda}\left(H^{n-1}(F), h\right) \leq \sum_{\Sigma_{j}} J_{\lambda}\left(K_{j}, h_{j}\right)$.

Proof. (a) is clear from the preceding remark.

(b) and (c). The monodromy $h$ acts on the inclusion morphism (a), so $h-\lambda$ id acts too. On the right hand side term, this amounts to the action of $h_{i}$, respectively of $h_{i}-\lambda \mathrm{id}$, on $H^{n-1}\left(F_{i}\right)$, independently for each $i$. We know that $h_{i}$ commutes with $\nu_{i}$.

The study may be pursued in case of higher dimensional singular locus Sing $f$; this is work in progress jointly with Dirk Siersma [ST]. 


\section{Controlled attaching}

This is a study of the cell-attaching described at the end of Facts 2.1(b) in the case $\operatorname{dim} \operatorname{Sing} f=1$ and $(X, 0)=\left(\mathbb{C}^{n+1}, 0\right)$. We refer to the notations and results in $₫ 2$ ? let us recall that condition $(*)$ is fulfilled in this case.

We shall use, and therefore need to recall from [Ti1] the construction of relative thimbles associated to the pair $\left(F, F^{\prime}\right)$ which are adapted to the carrousel monodromy. The disk $D_{\eta} \times\{\gamma\}$ will be called carrousel disk.

Our study on the attaching of thimbles starts with the following 5 steps. We refer to Figure 1. The first 3 steps apply to a singular space $(X, 0)$ under the condition $\left(^{*}\right)$.

(1). Our assumptions imply that $F^{\prime}$ is a bouquet of $n-1$ spheres. The restriction of $f$ to the slice $l=\eta$ has only isolated singularities: the intersections with $\operatorname{Sing} f$ and the intersections with $\Gamma$ (which are of Morse type, by the genericity of $l$ ). Therefore this is a deformation of the singularity $f_{\mid l=0}$ which is a partial Morsification.

(2). The result of attaching to $F^{\prime}$ the thimbles corresponding to the singularities in the zone $(l, f)^{-1}(\{\eta\} \times \delta)$ is, up to homotopy type, a bouquet of spheres of dimension $n-1$. The number of spheres is equal to the sum of the Milnor numbers of the singularities of the slice $\{f=0\} \cap\{l=\eta\}$. Now, remember from Facts 2.1(b) that we may identify the zone $(l, f)^{-1}(\{\eta\} \times \delta)$ to $(l, f)^{-1}(W)$, where $W$ is a certain open subset of the carrousel disk $D_{\eta} \times\{\gamma\}$ defined in [Ti1, §2].

The Milnor fiber $F:=(l, f)^{-1}\left(D_{\eta} \times\{\gamma\}\right)$ is obtained from $F^{\prime}:=(l, f)^{-1}(\eta, \gamma)$ by attaching a number of $n$-cells, each cell corresponding to one of the intersection points $\Gamma \cap F$. The total number of cells is equal to the intersection multiplicity $\operatorname{mult}_{0}(\Gamma,\{f=0\})$. We first attach to $F^{\prime}$ the thimbles from the zone $(l, f)^{-1}(W)$. We have seen before what is the result of this attaching.

(3). The carrousel model of the monodromy, introduced by Lê D.T. Lê2, together with the refined description of the carrousel monodromy from [Ti1], see [Ti1, Fig. 1, pag. 233], enables one to describe how the further attaching occurs.

If $\Delta$ is not empty, then there exist thimbles outside the zone $(l, f)^{-1}(W)$; this is due to the fact that all the components of $\Delta$ are tangent to the horizontal axis $\{f=0\}$.

Let us attach one "next" thimble, which is out of the zone $(l, f)^{-1}(W)$ ). By the main construction in [Ti1], this thimble is the image by the carrousel monodromy of a thimble from the zone $(l, f)^{-1}(W)$. Call the latter thimble $t^{0}$ and the former $t^{1}$. Say $t^{0}$ attaches to $F^{\prime}$ over the cycle $a$. By the construction in Ti1], the thimble $t^{1}$ will attach to $F^{\prime}$ exactly over the cycle $h_{1}(a)$, where $h_{1}$ denotes the geometric $f$-monodromy in the slice $F_{D}^{\prime}$ around the singular points $\operatorname{Sing} f \cap\{l=\eta\}$. All the above explanation is already contained in [Ti1], where the case $\operatorname{dim} \operatorname{Sing} f=0$ is treated; in that case the monodromy $h_{1}$ is geometrically trivial.

(4). What is $h_{1}(a)$ more precisely in the case $(X, 0)=\left(\mathbb{C}^{n+1}, 0\right)$ and $\operatorname{dim} \operatorname{Sing} f=1$ ? We consider the cycles up to homotopy equivalence and denote by $[\cdot]$ the homology or homotopy equivalence classes. In particular the additive notation for homotopy classes means that our homotopy groups are restricted to dimension $\geq 2$ and $\geq 3$ for relative homotopy groups (i.e. thimbles) respectively. 
Let us remark, firstly, that the $f$-monodromy in the slice $F_{D}^{\prime}$ around the singular points Sing $f \cap\{l=\eta\}$ splits into the direct sum of the monodromies around each of these points, since they are all in the same fibre of $f$. Secondly, that each such singularity may be more complex than a Morse singularity and so the monodromy around each of such singular points is the Coxeter element of some Morsification of the corresponding singularity. Then, by applying the Picard-Lefschetz rules to the monodromy $h_{1}$ we get:

$$
h_{1}([a])=[a]+\sum_{r} k_{r}\left[b_{r}\right]
$$

where $k_{r}$ is an integer and $b_{r}$ denotes one of the cycles vanishing at some point of Sing $f \cap$ $\{l=\eta\}$ (and where the sum is taken over these cycles).

(5). After all this discussion, we come back to the attaching of $t^{0}$ and $t^{1}$. Let $a$ be as chosen before. Notice now that the attaching of $t^{0}$ over $a$ just kills $a$, in other words $a$ is contractible in the space $F^{\prime} \cup(l, f)^{-1}(W)$. Next, the attaching of $t^{1}$ to $F^{\prime} \cup(l, f)^{-1}(W)$ is done over a cycle homotopy equivalent to $[a]+\sum_{r} k_{r}\left[b_{r}\right]$. Since $a$ is contractible in the space $F^{\prime} \cup(l, f)^{-1}(W)$, this new attaching is really over the cycle $\sum_{r} k_{r}\left[b_{r}\right]$. We have shown before that this cycle is not zero, and it is clearly independent on the cycles of type a. We get the following conclusion:

Proposition 4.1. Let $(X, 0)=\left(\mathbb{C}^{n+1}, 0\right)$ and $\operatorname{dim} \operatorname{Sing} f=1$. Assume that the linear function $l$ is sufficiently general. Then $F$ is obtained from $F^{\prime} \cup(l, f)^{-1}(W)$ by attaching thimbles defined by the carrousel monodromy, with attaching maps of the type $\sum_{r} k_{r}\left[b_{r}\right]$, where $b_{r}$ 's are the cycles of $F^{\prime}$ vanishing at the singular points $\operatorname{Sing} f \cap\{l=\eta\}$.

An exceptional case is when no thimbles are attached to $F^{\prime} \cup(l, f)^{-1}(W)$. This is equivalent to $\Delta$ being not tangent to the axis $f=0$, which can only happen if $\Delta=\emptyset$ for generic $l$, in which case one has $F \stackrel{\text { ht }}{\simeq} F^{\prime}$. This is in turn equivalent to the fact that the singular locus Sing $f$ is a line (by the non-splitting principle of Lê D.T.) and that the Milnor number of the transversal singularity is constant along the line. The equivalences can be deduced from the attaching results discussed in Facts 2.1.

Let us further remark that there exists a geometric cycle of type $a$ (i.e. a cycle over which attaches one of the carrousel thimbles from the zone $\left.(l, f)^{-1}(W)\right)$ which has nonzero intersection with at least one of the $b_{r}$ 's. This is due to the fact that the cycles of types $a$ and $b$ are together a basis of cycles (which one may arrange to be a geometric basis) of the isolated singularity at the origin of $f_{\mid l=0}$. Their intersection graph is connected, cf [La]. It is then an easy exercise* (which we may safely leave to the reader) to show that there exists at least one $\left[b_{r}\right]$ in the sum (11) such that its coefficient $k_{r}$ is non-zero.

The attaching map is precisely the boundary map $\partial: H_{n}\left(F, F^{\prime}\right) \rightarrow H_{n-1}\left(F^{\prime}\right)$ in homology. In homotopy, the additive notation has a meaning only if $n \geq 3$ and then the attaching map is the corresponding boundary morphism $\partial: \pi_{n}\left(F, F^{\prime} ;.\right) \rightarrow \pi_{n-1}\left(F^{\prime} ;.\right)$. Now remark that the monodromy $h$ acts on the exact sequence of the pair $\left(F, F^{\prime}\right)$, and its action on $H_{n-1}\left(F^{\prime}\right)$ or $\pi_{n-1}\left(F^{\prime}\right)$ is precisely $h_{1}$. Therefore $h_{1}(\partial(\alpha))=\partial(h(\alpha))$ for any linear combination $\alpha$ of carrousel thimbles. This proves the following, where coefficients are in some field: 
Corollary 4.2. Under the hypothesis of Proposition [4.1, assume in addition that the singular locus Sing $f$ is not a line with constant transversal Milnor number. Then the betti number $b_{n-1}(F)$ is strictly less than the sum $\sum_{i} b_{n-1}\left(F_{i}\right)$ of Milnor numbers of the singularities in the slice $\{l=\eta\} \cap\{f=0\}$.

This corollary has been announced independently by Lê D.T. and D.B. Massey [LM]. It is necessary to compare this result to Siersma's one in Si2 which coincides to Corollary [3.2(a) in case of a non-singular space germ $(X, 0)$. When Sing $f$ is a union of lines, then Corollary 4.2 provides a bound which might be better by at most one. Nevertheless in all other cases Siersma's result gives a better bound, or at least the same.

Corollary 4.3. Let $f$ define a line singularity with $A_{1}$-transversal generic singularity type and different from the transversal type at the origin. Let $[b]$ be the cycle vanishing at the $A_{1}$-transversal generic singularity. Then:

(a) In homology, there exists a linear combination of carrousel thimbles which attaches to $F^{\prime} \cup(l, f)^{-1}(W)$ with attaching map $[b]$.

(b) The Milnor fibre $F$ of $f$ is homotopy equivalent to a bouquet $\vee S^{n}$.

Proof. Let $\left[a_{i}\right]$ denote the cycles of $F^{\prime}$ which are killed by carrousel thimbles from the zone $(l, f)^{-1}(W)$. We claim that there exists a linear combination of carrousel thimbles $\left[t_{i}\right]$ such that the attaching map to $F^{\prime}$ is:

$$
\sum_{i} s_{i} h_{1}\left(t_{i}^{0}\right) \mapsto[b]+\sum_{i} s_{i}\left[a_{i}\right]
$$

where $s_{i} \in \mathbb{Z}$.

The claim follows from the following fact: if $p_{i}=\left\langle a_{i}, b\right\rangle$ denotes the intersection pairing then $\operatorname{gcd}\left\{\left|p_{i}\right|\right\}_{i}=1$. Indeed, if $v:=\operatorname{gcd}\left\{p_{i}\right\}_{i}>1$ then all $p_{i}$ 's are zero modulo $v$, which contradicts the fact that the Dynkin diagram of $f_{\mid l=0}$ relative to a distinguished basis is connected in the homology with coefficients any $\mathbb{Z}$-module (see e.g. [AGV, p. 77]).

Since $\operatorname{gcd}\left\{\left|p_{i}\right|\right\}_{i}=1$, there exist integer coefficients $s_{i}$ such that $\sum_{i} s_{i} p_{i}=1$. Therefore $\left\langle\sum_{i} s_{i}\left[a_{i}\right],[b]\right\rangle=1$. By Proposition 4.1 the linear combination of thimbles $\sum_{i} s_{i} h_{1}\left(t_{i}^{0}\right)$ attaches to $F^{\prime} \cup(l, f)^{-1}(W)$ with attaching map $\left\langle\sum_{i} s_{i}\left[a_{i}\right],[b]\right\rangle[b]$, which is homotopy equivalent to $[b]$. So this attaching will kill the cycle $[b]$.

(b). Part (a) implies that the reduced homology of $F$ is concentrated in dimension $n$. For $n \geq 3$ the above attaching result holds in homotopy too. We shall treat the cases $n=1,2$ separately.

For any $n \geq 2$ and in particular for $n=2$, we may apply a result by Lê-Saito [LS]: this says that in our situation the fundamental group of $F$ is abelian, hence trivial (since $H_{1}(F)=0$ ). Then $F$ has the homotopy groups of a bouquet $\vee S^{n}$, via the Hurewicz map, and therefore, by the Whitehead theorem for CW-complexes, it is homotopy equivalent to $\vee S^{n}$. As for the case $n=1, F$ has the homotopy type of a connected 1-dimensional CW-complex, hence $F \stackrel{\text { ht }}{\simeq} \vee S^{1}$.

Corollary 4.3(b) recovers, with a different proof, Siersma's bouquet result [Si1]. 


\section{REFERENCES}

[AGV] V.I. Arnol' d, S.M. Guseǔn-Zade, A.N. Varchenko, Singularities of differentiable maps, Vol. II: Monodromy and asymptotics of integrals, Monographs in Mathematics, 83. Birkhuser Boston, Inc., Boston, MA, 1988.

[GM] M. Goresky, R. MacPherson, Stratified Morse theory, Ergebnisse der Mathematik und ihrer Grenzgebiete. 3. Folge, Bd. 14. Berlin Springer-Verlag 1988.

[HL] H.A. Hamm, Lê D.T., Rectified homotopical depth and Grothendieck conjectures, The Grothendieck Festschrift, Vol. II, 311-351, Progr. Math., 87, Birkhuser Boston, Boston, MA, 1990.

[La] F. Lazzeri, A theorem on the monodromy of isolated singularities, Singularits Cargse (Rencontre Singularits Gom. Anal., Inst. tudes Sci. de Cargse, 1972), pp. 269-275. Asterisque, Nos. 7 et 8 , Soc. Math. France, Paris, 1973.

[Lê1] Lê D.T., Calcul du nombre de cycles évanouissants d'une hypersurface complexe, Ann. Inst. Fourier (Grenoble) 23 (1973), no. 4, 261-270.

[Lê2] Lê D.T., The geometry of the monodromy theorem, in: C. P. Ramanujam - a tribute, pp. 157-173, Tata Inst. Fund. Res. Studies in Math., 8, Springer, Berlin-New York, 1978.

[LM] Lê D.T., D.B. Massey, Hypersurface singularities and the swing, math.AG/0411432

[LS] Lê D.T., K. Saito, The local $\pi_{1}$ of the complement of a hypersurface with normal crossings in codimension 1 is abelian, Ark. Mat. 22 (1984), no. 1, 1-24.

[Lo] E.J.N. Looijenga, Isolated Singular Points on Complete Intersections, LMS Lecture Notes 77, Cambridge Univ. Press 1984.

[Mi] J. Milnor, Singular points of complex hypersurfaces, Ann. of Math. Studies 61, Princeton 1968.

[Si1] D. Siersma, Isolated line singularities, in: Singularities, Part 2 (Arcata, Calif., 1981), 485-496, Proc. Sympos. Pure Math., 40, Amer. Math. Soc., Providence, RI, 1983.

[Si2] D. Siersma, Variation mappings on singularities with a 1-dimensional critical locus, Topology 30, no. 3 (1991), 445-469.

[Si3] D. Siersma, The monodromy of a series of hypersurface singularities, Comment. Math. Helv. 65 (1990), no. 2, 181-197.

[ST] D. Siersma, M. Tibăr, Monodromy of nonisolated singularities, manuscript in progress

[St] J.H.M. Steenbrink, The spectrum of hypersurface singularities, Actes du Colloque de Théorie de Hodge (Luminy, 1987). Astérisque 179-180 (1989), 11, 163-184.

[Ti1] M. Tibăr, Bouquet decomposition of the Milnor fiber, Topology 35 (1996) 227-241.

[Ti2] M. Tibăr, A supplement to the Iomdin-Lê theorem for singularities with one-dimensional singular locus, Singularities and differential equations (Warsaw, 1993), 411-419, Banach Center Publ., 33, Polish Acad. Sci., Warsaw, 1996.

[Va] J.P. Vannier, Familles un paramtre de fonctions analytiques lieu singulier de dimension un, C. R. Acad. Sci. Paris Sr. I Math. 303 (1986), no. 8, 367-370.

(*) Solution of the exercise. Take as the order of the cycles indexed by $r$ 's the order of composing them in the Coxeter element. Then to the first index $r$ such that $\left\langle a, b_{r}\right\rangle \neq 0$ will correspond the coefficient $k_{r}= \pm\left\langle a, b_{r}\right\rangle$. The following $k_{r}$ 's are, in general, linear combinations of intersection numbers.

Mathématiques, UMR 8524 CNRS, Université des Sciences et Technologies de Lille, 59655 Villeneuve D'AscQ, France.

E-mail address: tibar@math.univ-lille1.fr 